\title{
ARCTiC LawE: An Upper-Body Exoskeleton for Firearm Training
}

\author{
Thomas M. Schnieders $^{1} \cdot$ Richard T. Stone $^{1} \cdot$ Tyler Oviatt $^{1} \cdot$ Erik Danford-Klein $^{1}$
}

Received: 1 July 2016/Accepted: 2 January 2017/Published online: 9 January 2017

(C) Springer SBM Singapore Private Ltd. 2017

\begin{abstract}
The Armed Robotic Control for Training in Civilian Law Enforcement, or ARCTiC LawE, is an upperbody exoskeleton designed to assist civilian, military, and law enforcement personnel in accurate, precise, and reliable handgun techniques. This exoskeleton training utilizes a laser-based handgun with similar dimensions, trigger pull, and break action to a Glock ${ }^{\circledR} 19$ pistol, common to both public and private security sectors. The paper aims to train and test subjects with no handgun training/experience both with and without the ARCTiC LawE and compare the results of accuracy, precision, and speed. Ultimately, the exoskeleton greatly impacts sensory motor learning, and the biomechanical implications are confirmed via both performance and physiological measurements. The researchers believe the ARCTiC LawE is a viable substitute for training with live-fire handguns in order to reduce the cost of training time and munitions. They also believe the ARCTiC LawE will increase accuracy and precision for typical law enforcement and military live-fire drills. Additionally, this paper increases the breadth of knowledge for exoskeletons as a tool for training.
\end{abstract}

Keywords Human-robot interaction - Exoskeletons · Biomechanics - ARCTiC LawE - Firearm training . Physical ergonomics

Thomas M. Schnieders

tms@iastate.edu

Richard T. Stone

rstone@iastate.edu

1 Iowa State University, 3027 Black Engineering, Ames, IA 50014, USA

\section{Introduction}

This paper proposes a design and evaluation of an upperbody exoskeleton for firearm training to assist civilian, military, and law enforcement in accurate, precise, and reliable handgun techniques. The Armed Robotic Control for Training in Civilian Law Enforcement, or ARCTiC LawE, for short, aims to train and test subjects with no handgun training/experience both with and without the ARCTiC LawE and compare the results of accuracy, precision, and speed. This upper-body exoskeleton training utilizes a laser-based handgun with similar dimensions, trigger pull, and break action to a Glock ${ }^{\circledR} 19$ pistol, common to both public and private security sectors. The laserbased firearm was chosen to ensure the safety of the participants as well as to alleviate any impact on bullet trajectories (as in traditional guns) due to humidity and/or temperature.

\section{Background}

Research has shown that tremors in the arm have negative effects on aiming [3-5, 8]; however, accuracy when aiming and firing a handgun depends on three primary factors: (1) environmental, (2) hardware, and (3) human factors [1]. A lot of devices have been developed to mitigate environmental impact and hardware impact on accuracy, but few exist to assist in training humans with handguns. The human factors that effect aim include: (1) fatigue [2], (2) experience [3, 4], (3) body sway [1], (4) heart rate [9], and (5) arm tremors [7].

Before the ARCTiC LawE, there was only one exoskeleton that is designed for handgun training-the MAXFAS, a mobile arm exoskeleton for firearm aim 
stabilization [7], designed and validated by Dan Baechle as part of his Master of Science research at the University of Delaware. Much of Baechle's research focused on manufacturing the exoskeleton out of carbon fiber and developing an algorithm that allowed for an intended motion while suppressing natural tremors.

The MAXFAS is essentially a series of cuffs, tension sensors, motors, and cables mounted to the exoskeleton and an aluminum frame above the user. Baechle's research utilized an airsoft pistol that uses a $\mathrm{CO}_{2}$ cartridge to replicate recoil and consisted of 20 participants aiming with an attached red laser. The end results of Baechle's experiments demonstrated that the MAXFAS, a cable-driven arm exoskeleton, is a viable method of improving pistol shooting performance.

Baechle provided possible limitations and potential future work as follows: (1) control mode limited with outdated motors, (2) tremor canceling algorithm should be tested on human subjects with new motors, (3) redesign of cuffs to reduce risk of pinching on participants' skin, (3) cabling should be routed through tubing, (4) increase participant pool with trained soldiers using a real pistol and aiming with the iron sights, (5) larger control group, (6) longer periods of shooting while wearing the exoskeleton, and (7) evaluate the effect of learning later than 5 min after removing the exoskeleton. The ARCTiC LawE can be seen as an extension of this work, in that it utilizes an exoskeleton for handgun training. The ARCTiC LawE forgoes a large aluminum frame that effectively keeps the participant stationary and uses only a glove, a gauntlet-like exoskeleton, and a small battery pack, allowing the participants to be much more mobile. Instead of $\mathrm{CO} 2$ cartridges and an airsoft pistol, the ARCTiC LawE uses an electronic handgun.

\section{Exoskeleton Design}

\section{Modeling the Human Arm}

As might be assumed, it was imperative to begin the design of the exoskeleton by first looking at the anthropometry of the human figure. Measurements for forearm length and breadth, the angle between the back of the hand and forearm, hand length (carpal to metacarpal bones), and handbreadth (across metacarpal bones) were taken from eight participants (four males, four females). In addition, the measurements of first to second knuckle length (proximal phalanges), second to third knuckle length (medial phalanges), and third knuckle to tip length (distal phalanges) were recorded for reach finger.

The measurements were divided into two groups:
- Group 1: 'small' hand/forearm sizes with handbreadth $6.9^{\prime \prime}-8.6^{\prime \prime}$.

- Group 2: 'large' hand/forearm sizes with handbreadth $8.9^{\prime \prime}-10.4^{\prime \prime}$.

These two groups formed the basis for the two sizes of exoskeleton gauntlets-medium and large. These participants were not used for physical testing of the ARCTiC LawE and served merely as a sampling of anthropometric sizes.

Anthropometric data were collected rather than consulting a table of anthropometry to look at the correlation of hand/forearm sizes and handbreadth to the angle formed by the back of the hand and the forearm when holding a handgun.

\section{Manufacturing the Exoskeleton}

By looking at traditional medieval gauntlets and patterns, new templates (which took into consideration the anthropometry data) were created on paper. The patterns were transferred from standard A4 printer paper to card stock and then cut out. The card stock templates were roughly folded along critical fold lines to match the principle investigator (PI). A second set was created to match the anthropometry of Group 2.

Placeholder rivet holes were cut out at approximate joint locations, keeping in mind the change of material from the much more flexible and forgiving card stock to stainless plate steel. Using a permanent marker, the cardstock template was traced onto $0.475-\mathrm{mm}, 26$-gauge, stainless plate steel. This stainless plate steel was relatively thin and was used as a rapid prototype for personal testing and verification of the template design (Fig. 1). The sheet metal was cut and formed by hand. The initial design made of thin sheet metal verified the proper design; however, a better method was required in order to stop radial and ulnar deviation. The new method would need to lock out the movement of the hand in the ' $\mathrm{Y}$ ' direction. The thin sheet metal was relatively easy to bend by radial and ulnar deviation of the user.

The original cardstock template was transferred onto 1.984-mm, 14-gauge, stainless plate steel and machined to size. Upon retrieval of machined parts, they were filed and

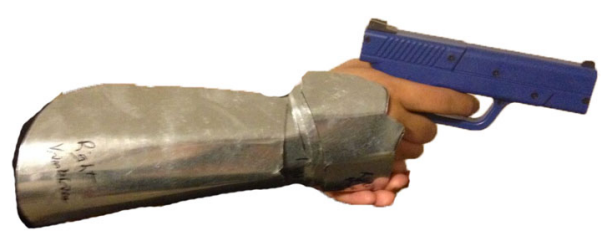

Fig. 1 ARCTiC LawE version 0.1 
deburred to ensure smooth edges. The parts were hand forged utilizing a series of blacksmith cold-forging techniques (i.e., dishing, die forming, raising, etc.) with multiple hammers and anvil-shaped objects. The finished coldforged exoskeleton sizes are shown in Fig. 2.

Based on the anthropometric chart, the individual metal parts were hammered into shape, first by dishing the underside using a ball peen hammer to create a proper semi-conical shape (narrower toward the wrist). Each part was roughly hammered to size with detail and precision in order to ensure each part fit as needed. The smooth edges that would function as overlapping plates were slightly bent a few degrees with a small hammer to ensure ease of sliding.

\section{How it Works}

For handguns, participants squeezed the trigger with the center of the tip of the index finger (distal phalanx). If participants squeezed the trigger with the outer tip of the finger, their shots erred to the left, whereas if participants squeezed the trigger with the inner portion of the finger, their shots erred to the right. In an effort to help guide participants in using the correct portion of their index finger, a neoprene glove, which also acts as padding between the user and the exoskeleton, had a portion of its index finger removed (Fig. 3). This allowed the participant to not only more easily feel the trigger, but also serve as a reminder for which portion of the finger should squeeze the trigger. Error is also caused by breaking the wrist up or

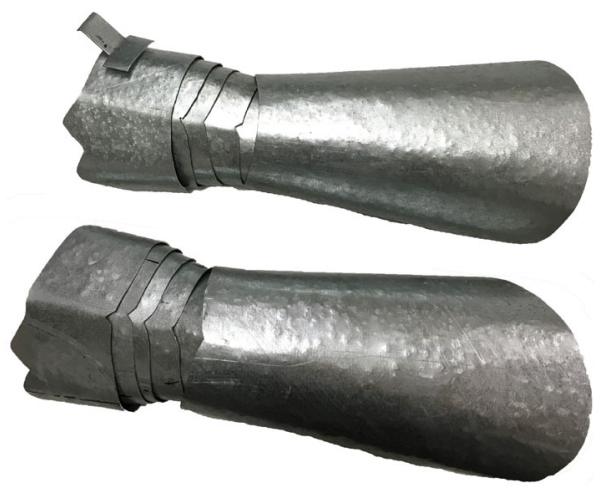

Fig. 2 Two sizes of exoskeleton

Fig. 3 Neoprene finger cutout

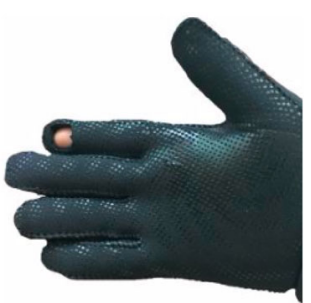

down, pushing, heeling, thumbing, etc., when handling the handgun, which causes the shot to fire up, down, left, right, and diagonally from the center of the target. Much of this has to do with: anticipating the recoil of the gun, pulling the trigger rather than squeezing it, or how the user is holding the grip of the gun.

The cut out portion of the neoprene glove serves to mitigate the effects of too little trigger finger and too much trigger finger, which results in hitting the target to the left and right of center, respectively. The stainless plate steel helps mitigate the breaking wrist up and down which results in hitting the target above and below center. To mitigate the tightening of the fingers or tightening of grip while pulling the triggers, Velcro was added to the pinky, ring, and middle finger in horizontal bars. Two bars of Velcro were sewn onto the proximal phalanges location of the neoprene glove, while one bar of Velcro was sewn onto the intermediate phalanges location of the neoprene glove. The two bars and one bar were used to help explain to the participant when matching up with the corresponding Velcro strips attached to the exoskeleton finger coupling.

The ARCTiC LawE is shown in Fig. 4. It shows the neoprene glove mated to the metal exoskeleton with the Velcro. The exoskeleton uses webbing that can easily be swapped out to accommodate multiple sizes. The webbing was held on with bolts, washers, and nuts to help facilitate swapping of the webbing. The finger coupling of the exoskeleton also acts as a guide for the participants. They were instructed to keep the Velcro on the neoprene glove mated with the exoskeleton helping mitigate over squeezing. The overlapping plates allow for some actuation in the flexion/extension of the wrist. This allows participants to easily draw and holster the LaserLyte training handgun during the experiment.

\section{Materials and Methods}

Participants were required to fill out a pre-study survey and sign an informed consent document. The pre-study survey asked participants their experience with guns, their experience with handguns, and questions regarding experience with video games and first-person shooters. Participants were comprised of civilians above the age of 18 who could

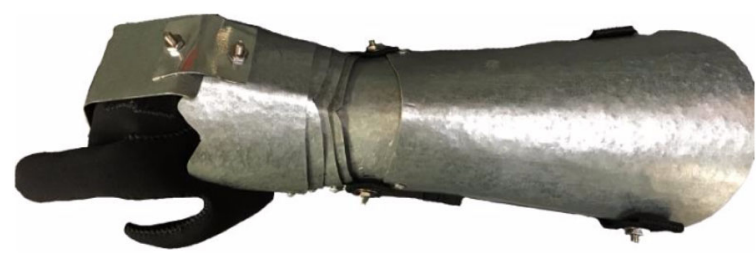

Fig. 4 ARCTiC LawE version 1 
legally give consent and could physically operate a handgun. Ideal participants had normal to corrected vision (contact lenses and glasses are okay except for bifocals, trifocals, layered lenses, or regression lenses) and had little to no experience using handguns.

Participants were randomly put into either a control group or experimental group. Training for both groups involved teaching participants' proper handgun usage and safety. While the study utilized a laser gun instead of live ammunition, participants were instructed to treat the laser gun as if it were a live gun using live ammunition. Examples of the handgun safety and proper use training included: always pointing the gun toward the ground until ready to fire, participants may not fire the laser gun unless anyone with them (i.e., the PIs) is behind them. Twenty participants originally signed up to participate in the study. However, from the data collected in the pre-study survey, four participants, all pre-allocated to the experimental group, self-identified as having moderate to advanced handgun experience. These four participants were removed from the study.

Participants were started at either 21 feet or 45 feet from the LaserLyte Score Tyme Board and then moved to the next distance to counteract the effect of learning on the results of the participants' scores. Participants were required to fire 25 shots at each distance for a total of 50 shots. The total score after the 25 th shot was recorded, and the target was reset. The testing was repeated for the remaining firing distance. Each distance had a potential for 250 points as a high score if each of the 25 shots hits the 10-point bull's-eye. The outermost ring of the target was worth four points, and rings incrementally increased by a value of one as you moved closer to the bull's-eye.

After completing the testing, participants filled out a post-study survey, which asked qualitative, self-identified metrics of perceived accuracy, perceived precision, etc.

\section{Results}

The participants were normally distributed. The $\mathrm{p}$ values support the hypothesis even further when noting that the control group had ten participants while the experimental only had six. The statistical significance threshold was set at 0.05 with practical significance set at 0.1 .

On average, the experimental group scored 64.9 points higher than the control at a 21-foot distance and 24.13 points higher than the control at a 45 -foot distance (Fig. 5). Among the participants in the experiment $(N=16)$, there was a statistically significant difference between the two groups at 21 feet, control $(\mathrm{M}=91.6, \mathrm{SD}=49.84)$ and experimental $(\mathrm{M}=156.5, \mathrm{SD}=23.83), t(15)=0.0018$, $p=0.01$. There was no statistically significant difference

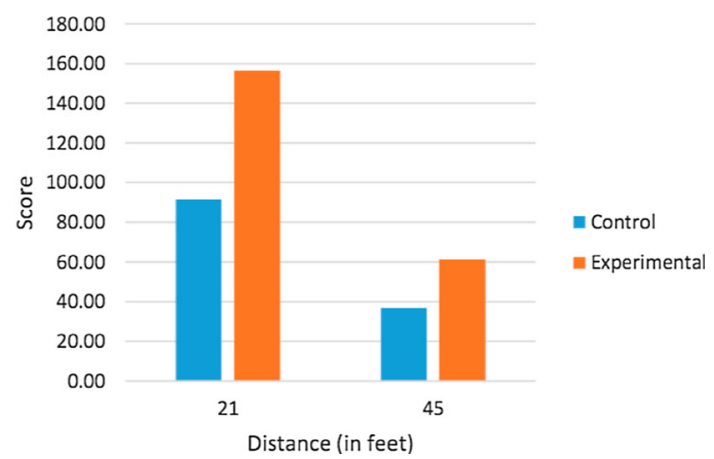

Fig. 5 Average score

between the groups at 45 feet, control $(\mathrm{M}=37.2$, $\mathrm{SD}=24.81)$ and experimental $(\mathrm{M}=61.33, \mathrm{SD}=35.81)$, $t(15)=0.09, p=0.13$.

In the post-study survey, participants were asked about the effectiveness of their training (Fig. 6), precision (Fig. 7), accuracy (Fig. 8), stability (Fig. 9), and opinion of how effective they thought the training would be over the course of 3 months. On average, participants in the experimental group rated their perceived effectiveness of the training 2.37 points (or $\sim 24 \%$ ) higher than the control group.

A statistically significant difference was found between the two groups, control $(\mathrm{M}=7.19, \mathrm{SD}=2.3)$ and experimental $(\mathrm{M}=8.67, \mathrm{SD}=0.82), t(15)=0.006, p=0.03$. On average, participants in the experimental group rated

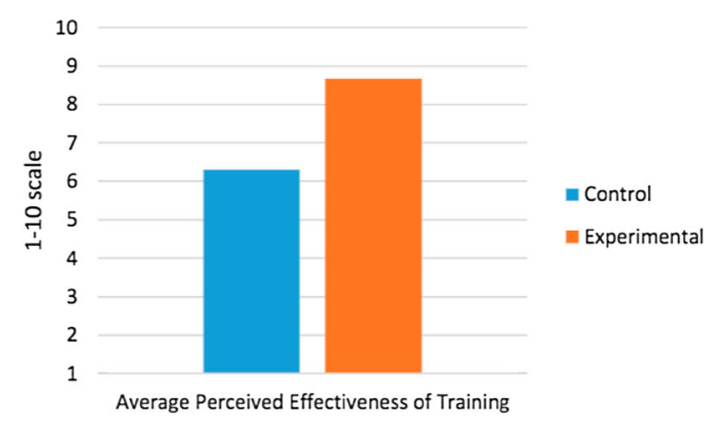

Fig. 6 Perceived effectiveness of training

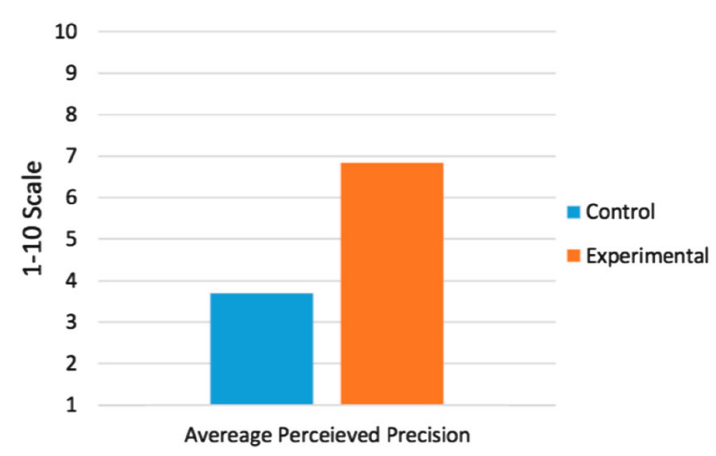

Fig. 7 Perceived precision 


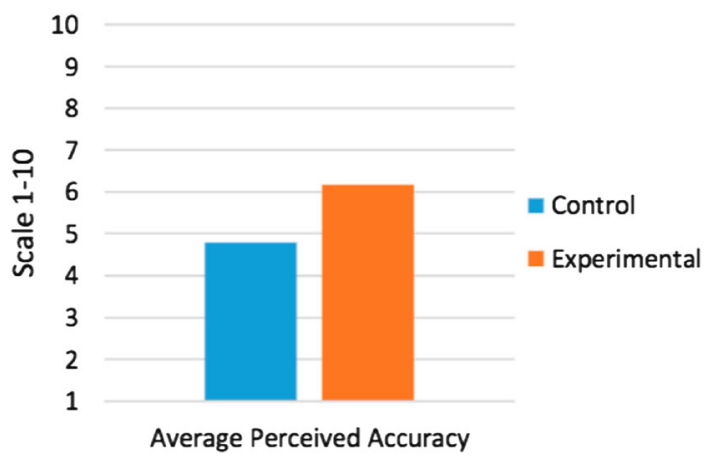

Fig. 8 Perceived accuracy

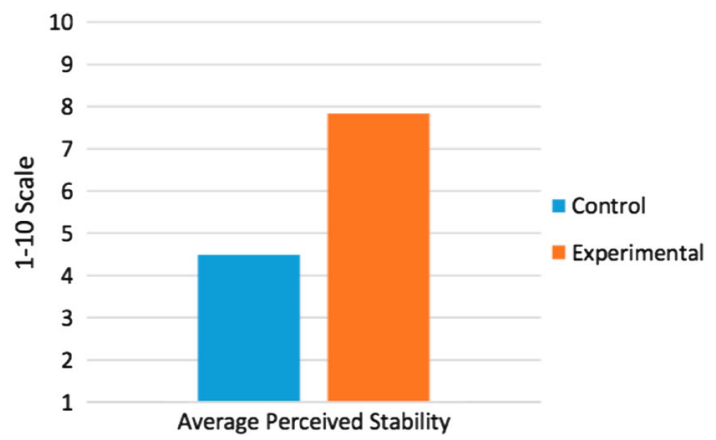

Fig. 9 Perceived stability

their perceived precision 3.13 points (or $\sim 31 \%$ ) higher than the control group. There was a statistically significant difference between the two groups, control $(\mathrm{M}=3.7$, $\mathrm{SD}=1.25)$ and experimental $(\mathrm{M}=6.83, \mathrm{SD}=1.17)$, $t(15)=0.00017, p<0.01$. On average, the experimental group rated their perceived accuracy 1.37 (or $\sim 14 \%$ ) higher than the control group. There was no statistically significant difference between the two groups, control $(\mathrm{M}=4.8, \quad \mathrm{SD}=1.87)$ and experimental $\quad(\mathrm{M}=6.17$, $\mathrm{SD}=1.60), t(15)=0.07, p=0.16$.

On average, the experimental group rated their perceived stability 3.33 (or $\sim 22.2 \%$ ) higher than the control group. There was a statistically significant difference between the two groups, control $(\mathrm{M}=4.5, \mathrm{SD}=1.65)$ and experimental $(\mathrm{M}=7.83, \mathrm{SD}=1.17), t(15)=0.00019, p<0.01$. On average, the experimental group scored 1.9 points (or $\sim 19 \%$ ) higher than the control group. It is important to note that this measure was taken in the post-study survey immediately following the study. There was a statistically significant difference between the two groups, control $(\mathrm{M}=7.1$, $\mathrm{SD}=1.91)$ and experimental $(\mathrm{M}=9, \quad \mathrm{SD}=1.26)$, $t(15)=0.16, p=0.049$.

The first study revealed enough statistical support for a second iteration which could address some of the qualitative results as well as the quantitative results. In particular, this first study showed fatigue from the participants attempting to 'rapid fire.' That is, the participants were

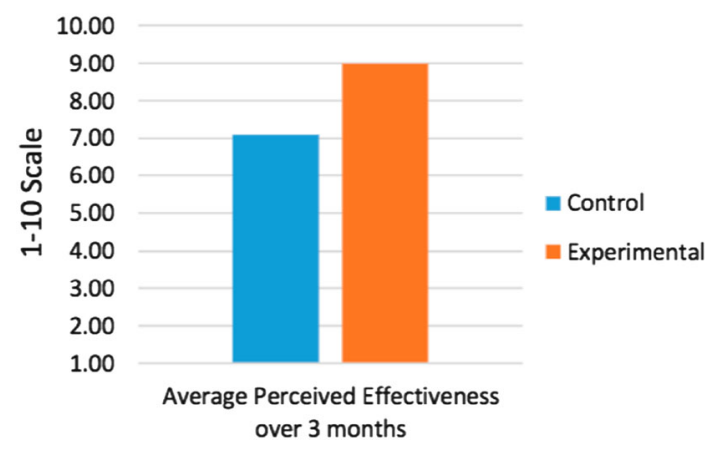

Fig. 10 Perceived effectiveness over 3 months

attempting to draw the LaserLyte, quickly fire the LaserLyte, holster the LaserLyte, and repeat in short order.

The results showed a tendency for participants to miss the target entirely, typically to the left or right of the target. If participants were hitting the target in the outermost ring, they would have a minimum score of 100 . This tells us that the exoskeleton needs to address wrist flexion and extension. Occasionally, participants would miss above or below the target, but this typically occurred within the first $10-15$ shots when participants with no handgun experience better learned how to aim with the handgun (Fig. 10).

\section{ARCTiC LawE version 2}

In order to address potential deflection to the left and right of the center of the target, caused by the extension and flexion of the wrist, a pull-type linear solenoid was used. The linear solenoid was attached to the gauntlet portion of the exoskeleton with a two-part epoxy. The solenoid was connected via a set screw and spring to the knuckle plate portion of the exoskeleton. The solenoid was turned on manually and powered by six AA batteries.

Moving out of the first study required testing of training effect. To do so, the participants in the second study were required to participate in the study on two separate days with approximately 1 week in between studies. Again, safety is always a primary concern when working with exoskeletons and humans. We use the padding of the neoprene glove again to provide a barrier between the metal (which has been filed down and deburred) and the user. The electrical components (solenoid, wiring, and battery pack) are a possible point of safety concern. However, this is addressed with proper care toward soldering the components and by using heat shrink wrap over any connection point ensuring safety to the participants. The second study looks at repeating the first study but utilizing the second version of ARCTiC LawE and includes 
a second week where participants are tested after having only been trained in week one.

\section{Participant Selection}

Similar to first study, students in one of the primary investigator's courses were invited to participate in the study for up to $5 \%$ extra credit in the class. Participants who emailed the PI asking to participate in one of the experiments for extra credit were compiled into a list and randomly assigned to experiments. Nineteen students were selected to participate in the second ARCTiC LawE study. The nineteen participants were randomly assigned to either the control group or the experimental group. The experimental group had ten participants, and the control group had nine participants.

Participants were comprised of civilians above the age of 18 who could legally give consent and could physically operate a handgun. Ideal participants had normal to corrected vision (contact lenses and glasses were okay except for bifocals, trifocals, layered lenses, or regression lenses) and had little to no experience using handguns. Again, some participants, after filling out the pre-study survey, self-identified as having moderate to significant handgun experience. These four participants were removed from the study. An additional two participants were removed for not responding to the scheduling poll, leaving a total of only thirteen participants. Two more participants were removed from the data set due to environmental factors during the testing that negatively impacted their scores. Both of these participants showed clear visible stress during the incidences, thus leaving only eleven participants for the second study. The experimental group had six participants, and the control group had five participants.

\section{Before Beginning the Experiment}

The rest of the methodology for study two, day one is the same as the first study with the following exceptions: (1) participants were not required to draw the LaserLyte handgun from the holster in study two, and (2) study two, week one used the second version of the ARCTiC LawE, Fig. 11.

\section{Second Study Day Two}

The second portion of the study took place approximately 1 week after the original training. Participants were not retrained, but were asked to fire at the two distances (starting at a different distance than their first study). This time, both the control and the experimental groups were tested without the exoskeleton and were asked to fill out the same post-study survey.
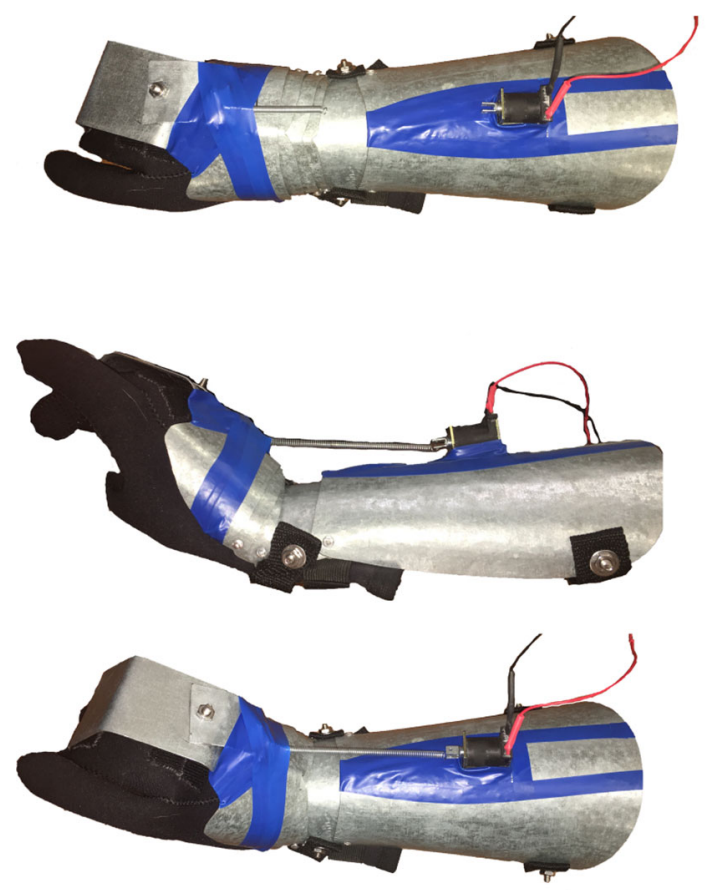

Fig. 11 ARCTiC LawE version 2. (Top) top-down view-unactuated. (Middle) side view-actuated. (Bottom) top-down view-actuated

\section{Week One}

On average, at a distance of 21 feet, the experimental group scored 37.1 points higher than the control group. There was not a statistically significant difference between the groups at 21 feet, control $(\mathrm{M}=70.4, \mathrm{SD}=52.35)$ and experimental $(\mathrm{M}=107.5, \mathrm{SD}=65.99), t(10)=0.16, p=0.34$. At a distance of 45 feet, the experimental group scored an average of 25.06 points higher than the control group. There was not a statistically significant difference between the two groups at 45 feet, control $(\mathrm{M}=28.6, \mathrm{SD}=12.36)$ and experimental $(\mathrm{M}=53.67, \mathrm{SD}=51.11), t(10)=0.15$, $p=0.32$. The experimental group, on average, rated the effectiveness of the training with the exoskeleton 1.6 points $(\sim 16 \%)$ higher than the control group's training without the exoskeleton. There was a statistically significant difference between the groups, control $(\mathrm{M}=6.4, \mathrm{SD}=1.14)$ and experimental $(\mathrm{M}=8, \quad \mathrm{SD}=1.1), t(10)=0.022$, $p=0.04$.

The experimental group, on average, rated their perceived precision 0.97 points $(\sim 9.7 \%)$ higher than the control group. There was no statistically significant difference between the groups, control $(\mathrm{M}=3.2, \mathrm{SD}=1.79)$ and experimental $(\mathrm{M}=4.17, \quad \mathrm{SD}=2.32), t(10)=0.228, p=0.47$. The experimental group rated their perceived accuracy 1.63 points $(\sim 16.3 \%)$ higher than the control group. This result was no statistically significant difference between the groups, control $(\mathrm{M}=2.2, \mathrm{SD}=1.64)$ and experimental $(\mathrm{M}=3.83, \mathrm{SD}=1.94), t(10)=0.083, p=0.17$. 
The experimental group rated their perceived stability with the exoskeleton 1.6 points $(\sim 16 \%)$ higher than the control group. There was no statistically significant difference between the groups, control $(\mathrm{M}=4.4, \mathrm{SD}=1.82)$ and experimental $(\mathrm{M}=6, \mathrm{SD}=1.67), t(10)=0.084$, $p$ value $=0.16$. The experimental group perceived the effectiveness of the exoskeleton training over a period of 3 months 0.123 points $(\sim 1.2 \%)$ lower than the control group. There was no statistically significant difference between the groups, control $(\mathrm{M}=8.8, \mathrm{SD}=1.79)$ and experimental $(\mathrm{M}=8.67, \quad \mathrm{SD}=1.21), \quad t(10)=0.445$, $p=0.89$.

\section{Week Two}

On average, at a distance of 21 feet, the experimental group scored 72 points higher than the control, and at a distance of 45 feet, the experimental group scored 14.7 points higher than the control group. There was no statistically significant difference between the groups at 21 feet, control $(\mathrm{M}=72.2, \mathrm{SD}=52.31)$ and experimental $(\mathrm{M}=124.17$, $\mathrm{SD}=43.03), t(10)=0.057, p=0.10)$. There was no statistically significant difference between the groups at 45 feet, control $(\mathrm{M}=47.8, \mathrm{SD}=27.14)$ and experimental $(\mathrm{M}=62.5, \mathrm{SD}=34.39), t(10) 0.224, p=0.46$ (Figs. 12, $13,14,15,16,17,18,19,20,21,22,23)$.

The experimental group perceived the effectiveness of the training only 0.5 points (or $5 \%$ ) higher than the control group. There was no statistically significant difference between the groups, control $(\mathrm{M}=6, \mathrm{SD}=1)$ and experimental $(\mathrm{M}=6.5, \mathrm{SD}=1.76), t(10)=0.29, p=0.59$. The experimental group rated their perceived precision 1 point $(\sim 10 \%)$ higher than the control group. There was no statistically significant difference between the groups, control $(\mathrm{M}=4, \mathrm{SD}=2)$ and experimental $(\mathrm{M}=5$, $\mathrm{SD}=2.19), t(10)=0.22, p=0.45$. The experimental group rated their perceived accuracy 0.83 points $(\sim 8.3 \%)$

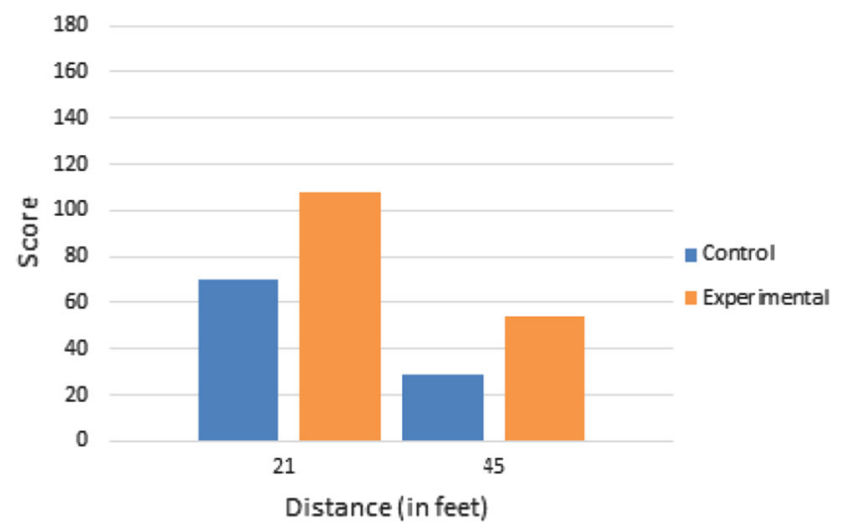

Fig. 12 Average score—study two week one

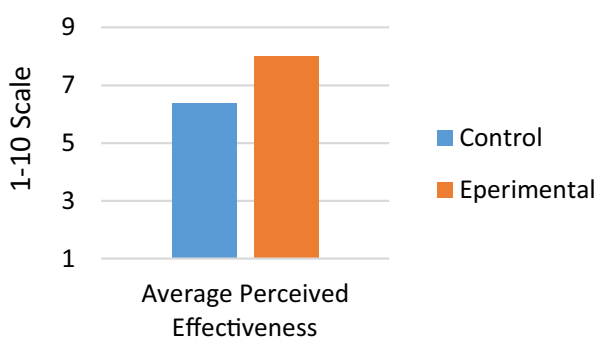

Fig. 13 Average perceived effectiveness-study two week one

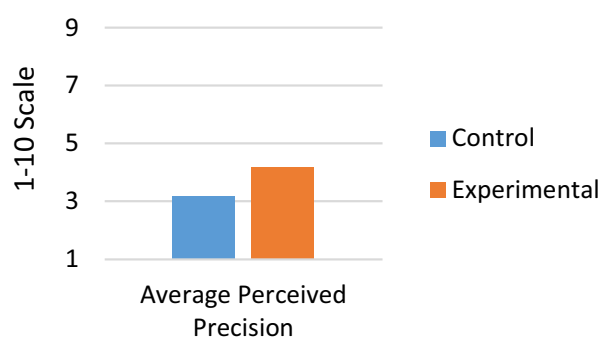

Fig. 14 Average perceived precision-study two week one

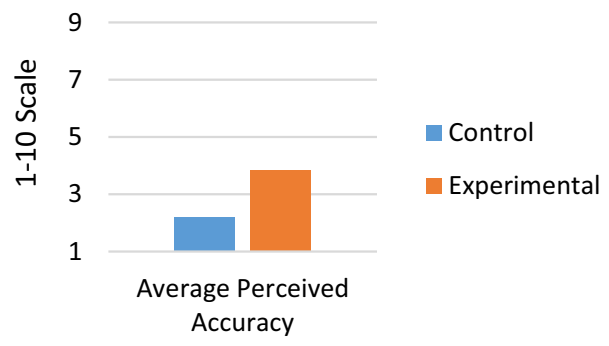

Fig. 15 Average perceived accuracy—study two week one

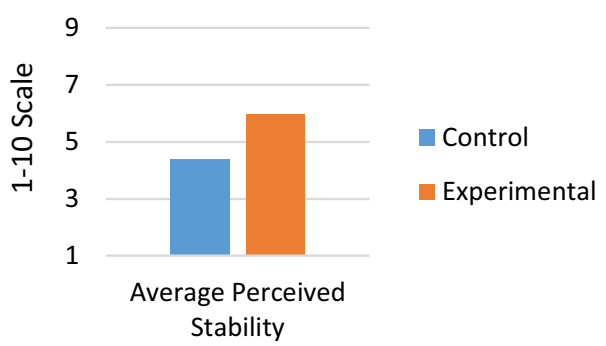

Fig. 16 Average perceived stability—study two week one

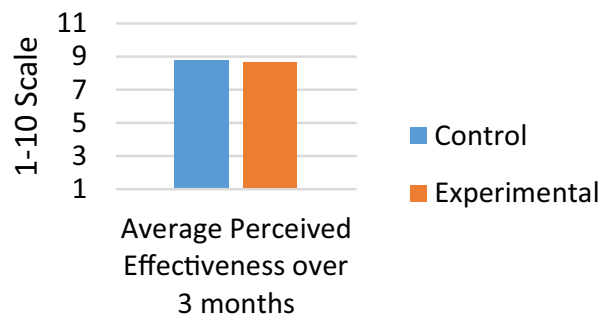

Fig. 17 Average perceived effectiveness over 3 months-study two week one 


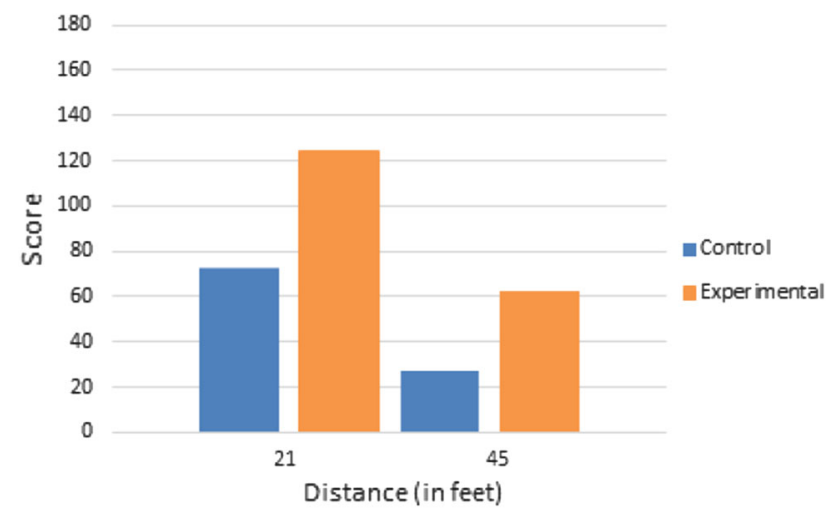

Fig. 18 Average score-study two week two

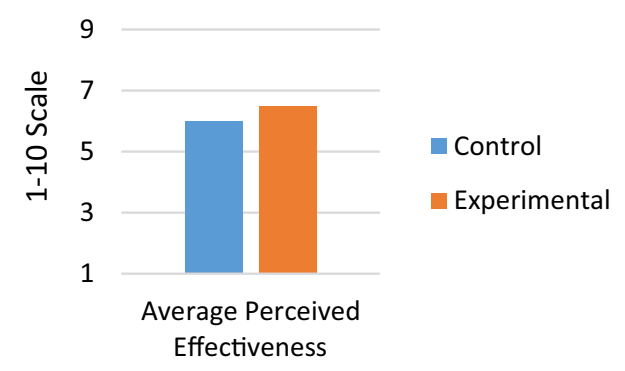

Fig. 19 Average perceived effectiveness-study two week two

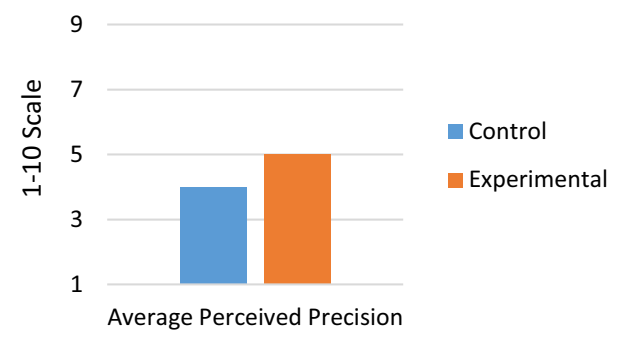

Fig. 20 Average perceived precision—study two week two

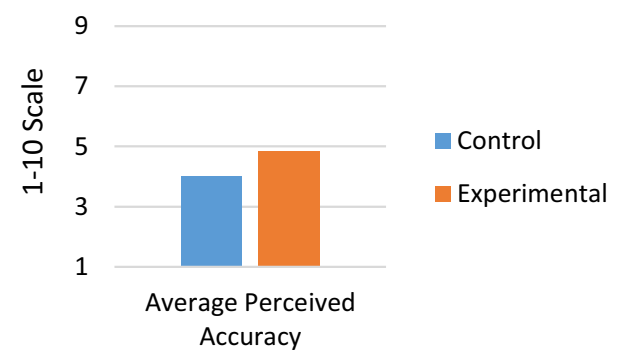

Fig. 21 Average perceived accuracy—study two week two

higher than the control group. There was no statistically significant difference between the groups, control $(\mathrm{M}=4$, $\mathrm{SD}=1.41)$ and experimental $(\mathrm{M}=4.83, \mathrm{SD}=1.72)$, $t(10)=0.20, p=0.41$.

The experimental group rated their perceived stability 1 point $(\sim 10 \%)$ higher than the control group. There was no

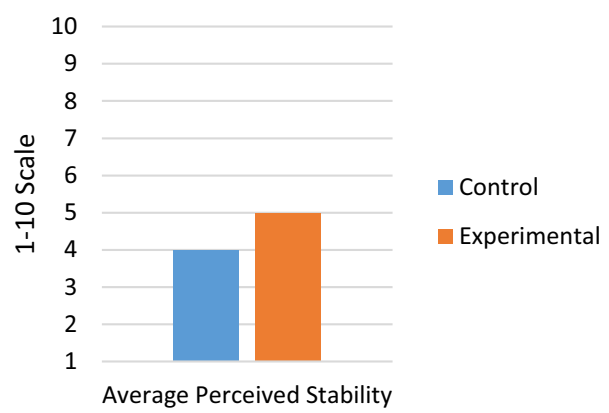

Fig. 22 Average perceived stability—study two week two

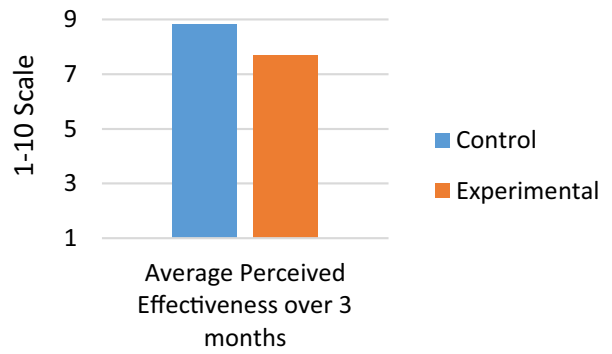

Fig. 23 Average perceived effectiveness over 3 months—-study two week two

statistically significant difference between the groups, control $(\mathrm{M}=4, \mathrm{SD}=1.22)$ and experimental $(\mathrm{M}=5$, $\mathrm{SD}=2), t(10)=0.17, p=0.36$.

The experimental group rated their perceived the effectiveness of the training over the course of 3 months to be 1.13 points $(\sim 11.3 \%)$ lower than the control group. There was no statistically significant difference between the groups, control $(\mathrm{M}=8.8, \mathrm{SD}=1.79)$ and experimental $(\mathrm{M}=7.67, \mathrm{SD}=1.75), t(10)=0.16, p=0.32$.

\section{Transfer of Training}

It is at this stage where transfer of training can be analyzed. The performance limiting factor is the retrieval from one's long-term memory. There are two types of knowledge that correspond to learning and training: (1) procedural and (2) declarative. The critical processes involved in cognitive learning are attention, rehearsal in working memory, retrieval from long-term memory, and metacognitive monitoring. Instructional technology directs cognitive learning processes. Augmented reality and virtual reality have been proven to be effective instructional technologies with the ability to display a transfer of training demonstrated in previous work with their use of virtual reality integrated weld training [6,7].

It can be stated that with respect to average score, the experimental group outperformed the control group with and without the ARCTiC LawE exoskeleton and a potential 
exists for a transfer of learning aspect. Future work would look at this aspect more in depth.

\section{Discussion}

Some future work includes replacing the manual activation of the solenoid with a gyroscope, which would automatically activate when the shooter's arm is in a firing position.

The transfer of training paradigm has a training effective ratio (TER) which is used to determine the transfer result of two or more groups - a control group using traditional technology and the experimental group using new technology. There are two possible transfer results: (1) negative transfer, where the experimental groups' performance is inferior to that of the control group and (2) positive transfer, where the experimental groups perform as well or better than the control group.

The training effectiveness ratio is as follows:

$\frac{Y_{c}-Y_{x}}{Y_{c}}$

where $Y_{c}$ is the time, trials, or errors required by a control group to reach a performance criterion and $Y_{x}$ is the corresponding value for an experimental, or transfer, group having received prior practice on another task.

For future studies, the group trained with the LaserLyte handgun would be the control group and the group trained with the ARCTiC LawE and the LaserLyte handgun would be the experimental group. The amount of time was not recorded for the first and second study. However, it was noted that no appreciable difference existed in regard to training time between the control group and the experimental group.

While future studies that look more in-depth at the TER may be required, it is important to note that the studies involved with the ARCTiC LawE gave much more time between training and re-testing than the MAXFAS exoskeleton. Participants in the second ARCTiC LawE study had a week long gap between training and testing, whereas the MAXFAS exoskeleton study (involving five control participants and fifteen experimental participants) gave only a 5-min gap. The future work here would include determining the appropriate score for a qualified police officer and comparing the LaserLyte training with the training with the ARCTiC LawE. An additional task would be to compare the TER with a traditional handgun over a full training period.

Some potential future work includes changing what material of the exoskeleton. A change from the 14-gauge stainless steel to fiberglass would reduce the weight while maintaining the rigidity and structural integrity of the exoskeleton. Another possible replacement material would be $3 \mathrm{D}$ printed $\mathrm{ABS}$ plastic. This material would also reduce the weight without compromising the structural integrity of the exoskeleton. This would also allow for parts that could be quickly and cheaply replaced or swapped out for smaller or larger parts or swapped out for specialized equipment.

Another point of potential future work, based on advice from military personnel, would be to include different training routines that involve testing a Weaver stance where the non-dominant foot is in the forward position instead of the dominant foot; including walking drills (forward and/or sideways) to test the effectiveness of mobile training and rapid response time; including moving targets; and to look at integrating the exoskeleton not only for handguns but also as a tool for rifle training.

Multiple military personnel whose data were excluded from the study really liked the idea of the exoskeleton and originally thought it was designed as an everyday carry piece of equipment. One individual stated that they would be willing to purchase the exoskeleton for everyday carry. They initially thought it was a little cumbersome and heavy, but after running through the study, stated that they barely felt it on their arm. The same individual stated that the exoskeleton helped them stabilize their shooting arm. They stated that they had to worry less about stabilizing their arm and could focus more on aiming at their target. When the military personnel were informed that it was not originally designed as an everyday carry but rather as a training tool for novice shooters, they were even more ecstatic and enthusiastic about the paper.

The following extrapolation is made from the assumption that other environmental aspects like sound are not major factors.

$Y\left(x^{*}\right)=\frac{y_{k-1}+\left(x^{*}-x_{k-1}\right)}{x_{k-x} * y_{k-y_{k-1}}}$

A document released by the U.S. Department of Homeland Security covers the ammunition usage and purchase history for fiscal years 2010-2012 and is summarized in Table 1.

Buying 0.40 S\&W 180 grain full metal jacket rounds in bulk (cheaper than when buying fewer rounds) costs $\$ 120$ for 500 rounds [1] or about $\$ 0.24$ each. Based on the information above, it can be expected that tor the 2016 fiscal year, the Department of Homeland Security will have spent $\sim \$ 6.4 \mathrm{M}$ just on the bullets for training. From discussions with Dr. Richard T. Stone, a Reserve Deputy in Story County, as well as other police officers during the

Table 1 U.S. DHS ammunition usage and spending FY 2010-2012 [10]

\begin{tabular}{ll}
\hline FY 2010 & $148,314,825$ bullets \\
FY 2011 & $108,664,054$ bullets \\
FY 2012 & $103,178,200$ bullets \\
\hline
\end{tabular}


PI's initial training with handguns, it was found that there is a decrease in purchasing of ammunition and an increase in the cost per bullet each year, for various reasons. Even with the decreasing supply and increasing costs, servicemen and servicewomen cannot afford to not be at an appropriate level of training and the LaserLyte and ARCTiC LawE can be a viable supplement for traditional training.

Even a small decrease in cost of ammunition, which can be experimentally determined with the comparison of the ARCTiC LawE training to live-fire training, can result in a large amount of savings. This would greatly reconcile any initial investment cost. This does not include any money saved on training personnel.

It is typical for police officer training to spend 40-h weeks on firearms training, requiring approximately 1000 rounds of 0.40 caliber rounds per week. Forty hours is a minimum amount of training required to carry a handgun in the USA.

Based on results of transfer of training with virtual reality and welding [6,7], and based on discussion with the local Sheriff's department, a reduction in number of bullets needed to train police officers of $50 \%$ could be considered a conservative amount. While real-world application and virtual application are not a direct comparison, it has been proven to provide a positive transfer of training and is something that could be done in the future.

\section{Conclusion}

Ultimately, the exoskeleton greatly impacts sensory motor learning, and the biomechanical implications are confirmed via both performance and physiological measurements. The researchers believe the ARCTiC LawE to be a viable substitute for training with live-fire handguns to reduce the cost of training time and munitions and will increase accuracy and precision for typical law enforcement and military live-fire drills. This paper increases the breadth of knowledge for exoskeletons as a tool for training.

\section{References}

1. Baechle DM (2013) MAXFAS: a mobile arm exoskeleton for firearm aim stabilization. Master's thesis, University of Delaware

2. Fröberg JE, Karlsson C, Levi L, Lidberg L (1975) Circadian rhythms of catecholamine excretion, shooting range performance and self-ratings of fatigue during sleep deprivation. Biol Psychol 2(3): $175-188$

3. Goontilleke RS, Hoffmann ER, Lau WC (2009) Pistol shooting accuracy as dependent on experience, eyes being opened and available viewing time. Appl Ergon 40(3):500-508

4. Kuribayashi K, Shimizu S, Okimura K, Taniguchi T (1993) A discrimination system using neural networks for EMG-control prostheses-integral type of EMG signal processing. In: Proceedings of 1993 IEEE/RSJ international conference on intelligent robots and systems, pp 1750-1755

5. Mizen NJ (1965) Preliminary design for the shoulders and arms of a powered, exoskeletal structure. Cornell Aeronautical Laboratory Report VO-1692-V-4

6. Stone RT, McLaurin E, Zhong P, Watts K (2013) Full virtual reality vs. integrated virtual reality training in welding. Welding 92(6): $167 \mathrm{~s}-174 \mathrm{~s}$

7. Stone RT, Watts KP, Zhong P (2011) Virtual reality integrated weld training. Welding 90(7):136-137

8. Singh RM, Chatterji S (2012) Trends and challenges in EMG based control scheme of exoskeleton robots-a review. Int J Sci Eng Res 3(8)

9. Tharion WJ, Santee WR, Wallace RF (1992) The influence of heart rate, rectal temperature and arm-hand steadiness on rifle marksmanship during and after field marching in MOPP 0 and MOPP I. U.S. Army Research Laboratory, Aberdeen Proving Ground, MD

10. Usage and Purchase History of Ammunition Provided by the Department of Homeland Security. Congressman Billy Long.Web. 31 March 2016 